\title{
On the Particles Size Distributions of Diatomaceous Earth and Perlite Granulations
}

\author{
Dragan V. Petrović1 - Časlav B. Mitrović2,* - Nataša R. Trišovic ${ }^{2}$ - Zorana Z. Golubovićc \\ 1 University of Belgrade, Faculty of Agriculture, Serbia \\ 2 University of Belgrade, Faculty of Mechanical Engineering, Serbia
}

\begin{abstract}
When filtering products with a high level of non-soluble solids and containing filtration-inhibiting substances, a variety of different filter aids can be used. Among others, kieselguhr and perlite are possible materials for this purpose. The selection of the granulation that should be applied depends on the filtered liquid medium and the desired retention level. While perlite is most commonly used for rough filtration, kieselguhr is also suitable for more subtile filtration. In the paper, particle size distributions of three kieselguhr granulations and a perlite granulation are analysed. Particle sizes are measured by the morphometric method, commonly used in the microbiology. Basic statistic parameters are calculated for all considered samples and compared. The applicability of hyperbolic and log-hyperbolic functions, in describing the particles size distributions of these granulations is verified. Depending on the imposed filtration requirements, this approach enables simple modelling and composition of a wide variety of different granulations characterised by appropriate particle size distributions from a few available granulations. (C)2011 Journal of Mechanical Engineering. All rights reserved.
\end{abstract}

Keywords: filtration, kieselguhr, perlite, granulation, particle size distribution

\section{INTRODUCTION}

Filtration of fluids is an important step in a variety of technological processes. There are several types of filtration. In order to optimise the filtration process, they can be applied either independently, or combined. However, one of the most often used is mechanical filtration [1]. In the filtration of this kind, substances are separated from each other by the application of adequate filtration mediums and procedures. In this way, contaminants are restrained at the surface, or inside the volume of the filtration medium. Depending on the initial and final process conditions, the filtration procedure can include one or more succeeding filtration steps. In the latter case, fluid is gradually filtered - primary filter performs rough filtration, while the last filtration step extracts the finest contaminants from the fluid.

To date, a variety of filtering mediums and filter aids have been developed and reported in a rich literature data base. The filtering medium has been fabricated as ceramics [2] and [3], polymers [4] and [5], metals [6] and [7], zeolite [8] and [9], celulose [10] and [11], carbon [11] and [12], composite materials [13] and [14], their combinations [15],[16] and [25], etc.
The frequently used filter aids materials are: kieselguhr (also known as diatomaceous earth - DE) and perlite, etc. Distributions of particle sizes of these two filter aid materials significantly influence the filtration process.

DE is a chalky sedimentary material, comprised of the skeletal remains of prehistoric water microorganisms (single-celled algae) [17], called diatoms. They are characterized by size in the range from under 5 to over $100 \mu \mathrm{m}$ and porous structure with openings as small as $0.1 \mu \mathrm{m}$ in diameter. The diatom skeletons and housings are mined, ground up and thermally treated to render a powder composed of microscopic solid particles, which occupy only $15 \%$ of total filtration volume. The combination of small pore sizes, large specific surface area, firmness of the particles and high porosity allow DE to efficiently remove small particles from liquid mediums at high filtration rates. The low solubility of DE in water and diluted acids (less than 1\%), as well as the odorless, tasteless, and chemically inert characteristics, make it safe for filtering water or other liquids intended for human consumption.

$\mathrm{DE}$ is commonly applied in different areas: medicine and bioprocessing [15] and [18], the food and beverage industry [19] to [21], purification of 
potable water, contaminated ground- and surface water [22] and [23], decontamination of sewage liquids and waste water [24], pharmacy [25], etc. However, there are some difficulties related to efficient recycling of DE and its expensive disposal due to increasing landfill taxes [22]. Fortunately, there are some promising attempts to further processing and using the DE sludge from deep filtration beds [19] and [24].

Perlite is an amorphous volcanic glass that has 2 to $5 \%$ of combined water. At temperatures of 760 to $1100{ }^{\circ} \mathrm{C}$, it softens. Water trapped in the structure of the material vaporizes and escapes, causing the expansion of the material [26]. Due to its low density after being processed, and relatively low price, many commercial applications for perlite have been developed, including the removal of: hazardous metals [27] and [28], dye pollutions, particulates from exhaust gases, medicine and bioprocessing [15] and [18], as well as in the food and beverage industry [11].

A vast variety of $\mathrm{DE}$ and perlite granulations exists. The selection of the applied granulation depends on the filtered fluid properties and the desired retention level. While perlit is most commonly used for rough filtration, DE is also suitable for more subtile filtration. However, these two materials are frequently combined in a common filtering process [11].

The particle size distributions of DE and perlite granulations, together with size distributions and concentration of solid contaminants in the dirty inlet and outlet purified fluid, directly influence deep filter properties and govern the preparation of adequate filtering mediums and aids. Consequently, these problems have been extensively studied worldwide.

In this paper, particle size distributions of three DE and one perlit granulation are analysed. Sizes of acquired particles are measured by the morphometric method. The applicability of hyperbolic and log-hyperbolic functions, in describing the particles size distributions of these granulations is verified. Depending on the filtration requirements, this approach facilitates modelling and composition of variety of different granulations characterised by appropriate particle size distributions from a few available granulations.

\section{MATERIAL AND METHODS}

The paper presents and analyses particles size distributions of three DE granulations (Pura, Super and Ultra -made by Pall SeitzSchenk), and a perlite granulation (Harbolite, type 900 produced by World Minerals). Distributions of their particle sizes are measured by the morpho-metric method. It is developed primarily for microbiological analysis, but it is quite effective and accurate for estimating the size of various microscopic objects including solid particles. Each granulation sample was separately mixed with distilled water - the so-called »dilution ratio« of $10^{-3}$. Concentration of the sample was performed with Pall filtration equipment, using $47 \mathrm{~mm}$ filter disc membranes whose absolute removal rating is 0.2 $\mu \mathrm{m}$. Proximately before counting the particles, filter discs were flushed by redistilled water, and obtained suspension was centrifuged to provide an appropriate concentration for measuring. Consequently, sizes of $\mathrm{DE}$ and perlite particles were measured. An analogue technique, also based on the application of optical microscope, can be used in the technical practice to research the flocs size distributions in the suspensions of biological water treatment plants.

Basic statistic parameters are calculated for each of the four samples following the common procedure. Particles of each sample are sorted according to their diameters in the intervals of $\Delta d=5 \mu \mathrm{m}$ in width. This way, each of $m$ particle size-classes is represented by the interval midpoint $d_{i}$ and the absolute frequency (number of particles) $N_{i}=N\left(d_{i}\right)$. Consequently, the sum of absolute frequencies equals the total sample particles number:

$$
N=\sum_{i=1}^{m} N_{i}=\sum_{i=1}^{m} N\left(d_{i}\right) .
$$

When the relative frequencies:

$$
Q_{0 i}=Q_{0}\left(d_{i}\right)=\frac{N_{i}}{N},(i=1,2, \ldots, m),
$$

of the particle sizes are evaluated, basic statistical parameters are calculated. Arithmetic mean and standard deviation:

$$
\bar{d}=\sum_{i=1}^{m} Q_{0 i} \cdot d_{i}
$$




$$
\sigma=\sqrt{\sum_{i=1}^{m} Q_{0 i} \cdot\left(d_{i}-\bar{d}\right)^{2}}
$$

represent an expected value and dispersion of data sample, respectively. Skewness $S$ and flatness $F$ factor:

$$
\begin{gathered}
S=\frac{\sum_{i=1}^{m} Q_{0 i} \cdot\left(d_{i}-\bar{d}\right)^{3}}{\sigma^{3}}, \\
F=\frac{\sum_{i=1}^{m} Q_{0 i} \cdot\left(d_{i}-\bar{d}\right)^{4}}{\sigma^{4}},
\end{gathered}
$$

characterise a distribution shape: symmetry and peakedness, respectively. For the Gaussian function $S=0$ and $F=3$. A higher difference between the empirical and these theoretical values of $S$ and $F$ means larger discrepancy of the empirical distribution according to the normal.

Characterisation of some distribution by different statistical description parameters provides useful information. However, additional approaches also exist - approximating a measured distribution with interpolation, spline and appropriate analytic fitting functions, [23] to [25]. The approach based on data fitting is used in the present paper. Its purpose is to characterize a large amount of information contained in the empirical distribution, with a small number of parameters constants in the probability distribution function $(P D F)$, evaluated with the fitting procedure.

In general, normal $P D F$ is the most commonly used statistical function. However, $P D F \mathrm{~s}$ of real samples very often significantly deviate from this function. Among others, a possible approach for overcoming these situations is based on the hyperbolic function, introduced by Barndorf-Nielsen (1977) to describe the asymmetric distributions of the wind-blown sand particle sizes [26] and has been extensively used up to date [27] and [28]. This method is used in the present paper for modelling the PDFs of filtering granulation particle sizes.

The probability density function:

$$
p d f(x)=\frac{d[P D F(x)]}{d x},
$$

can be represented by hyperbolic function in the following form:

$$
\begin{gathered}
p d f(x)=g(x)=A(\alpha, \beta, \delta) . \\
\cdot \exp \left[-\alpha \cdot \sqrt{\delta^{2}+(x-\mu)^{2}}+\beta \cdot(x-\mu)\right] .
\end{gathered}
$$

The four arbitrary parameters, $\alpha>0$, $|\beta|>\alpha, \delta>0$ and $\mu \in(-\infty, \infty)$ that define its shape are evaluated numerically on the basis of adequate empirical data set. Parameter $A$ is the normalization constant that ensures the hyperbolic function, Eq. (8), to satisfy the standard condition of the theory of probability, the so-called normalizing condition:

$$
\int_{-\infty}^{\infty} g(x) \cdot d x=\int_{-\infty}^{\infty} p d f(x) \cdot d x=1
$$

This means that the probability of all possible realizations of some event i.e. the probability of a sure event is $1(100 \%)$. This constant is defined by formula:

$$
\begin{gathered}
A(\alpha, \beta, \delta)=\frac{\sqrt{\alpha^{2}-\beta^{2}}}{2 \cdot \alpha \cdot \delta \cdot K_{1}(\xi)}, \\
\xi=\delta \cdot \sqrt{\alpha^{2}-\beta^{2}},
\end{gathered}
$$

where $K_{1}(\xi)$ is the Bessel function of the third kind and the first order. If a natural logarithm $\ln (x)$ is applied in Eq. (8) instead of $x$, the log-hyperbolic function arise.

To analyse some properties of the hyperbolic function, Eq. (8) can be written as:

$$
\begin{gathered}
\ln [g(x)]=\ln (A)- \\
-\alpha \cdot \sqrt{\delta^{2}+(x-\mu)^{2}}+\beta \cdot(x-\mu) .
\end{gathered}
$$

This functional form shows that two asymptotes exist in this situation, having slopes $(\alpha+\beta)$ and $-(\alpha-\beta)$. In addition, the parameter $\mu$ gives the abscissa of the point of intersection of the two asymptotes and is, therefore, regarded as a location parameter. The location $\mu$, the scale parameter $\delta$ and the mode point $v$ of the hyperbolic distribution are interrelated:

$$
v=\mu+\frac{\delta \cdot \beta}{\sqrt{\alpha^{2}-\beta^{2}}} .
$$

The radius of curvature at the mode point, 


$$
\rho_{v}=\frac{\alpha^{2} \cdot \delta^{2}}{\sqrt{\left(\alpha^{2}-\beta^{2}\right)^{3}}},
$$

characterises the distribution peakedness. For the normal function $\rho_{v}$ equals the variance $\sigma^{2}$.

Basic parameters of hyperbolic function are sketched in Fig. 1.

In general, the hyperbolic function possesses thicker tails in comparison to the normal Gaussian. It may be skewed to the left $(\beta<0)$, symmetric $(\beta=0)$ or right-skewed $(\beta>0)$. All these properties of the hyperbolic function are also illustrated in Fig. 1. Following [28], by giving parameters in the hyperbolic function certain suitable limits, it is possible to convert it to the normal (Gaussian) and Laplace (symmetrical or asymmetrical) distribution.

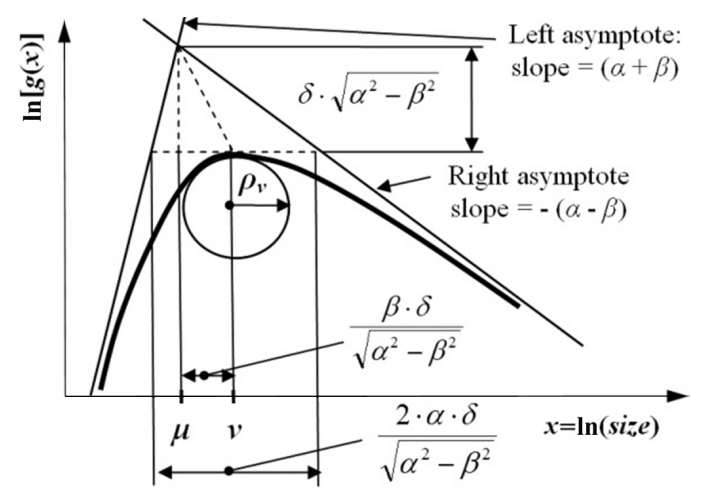

Fig. 1. Geometric interpretation of relevant parameters of hyperbolic function

For a known $p d f(x)$ of a specified granulation, where $x$ is a particle diameter $d$, the participation of the particles having diameters within limits $d_{\min }$ and $d_{\max }$ can be evaluated:

$$
P\left(d_{\min }<x<d_{\max }\right)=\int_{d_{\max }}^{d_{\min }} p d f(x) \cdot d x .
$$

A standard question that follows empirical data fitting procedures is related to their accuracy. The most commonly, fitting accuracy can be estimated by the error sum of squares, also called the residual sum of squares:

$$
S S E=\sum_{i=1}^{m}\left(y_{i}-\hat{y}_{i}\right)^{2}=\sum_{i=1}^{m} \varepsilon_{i}^{2}
$$

It represents a sum of squares of regression errors $\varepsilon_{i}$, i.e. the differences between the true (measured) values $y_{i}$ with respect to fitted values $y_{i}$, where $m$ is the number of fitted data "points «, equal to the number of particle size-class intervals in this case. The root mean square error, or standard error of estimate:

$$
R M S E=\sqrt{\frac{S S E}{m-k}}=\sqrt{\frac{\sum_{i=1}^{m}\left(y_{i}-\hat{y}\right)^{2}}{m-k}}=\sqrt{\frac{\sum_{i=1}^{m} \varepsilon_{i}^{2}}{m-k}},
$$

is the second popular characterization parameter of fitting accuracy. Deviations are referred to each predicted value. Thus, SSE has $m-4$ degrees of freedom if a regression line is hyperbolic, since $k=4$ degrees of freedom are lost: the four constants in the Eq. (8) are evaluated. The smaller values, defined by Eqs. (15) and (16), mean the higher fitting accuracy.

However, the most commonly used is the coefficient of determination or R-square:

$$
R^{2}=\frac{S S T-S S E}{S S T}=1-\frac{S S E}{S S T}=1-\frac{\sum_{i=1}^{m}\left(y_{i}-\hat{y}_{i}\right)^{2}}{\sum_{i=1}^{m}\left(y_{i}-\bar{y}\right)^{2}} \in[0,1],
$$

which represents the ratio between the residual sum of squares SSE, Eq. (15), and the total sum of squares:

$$
S S T=\sum_{i=1}^{m}\left(y_{i}-\bar{y}\right)^{2}
$$

where $\bar{y}$ denotes the mean value. As closer is the value of $\mathrm{R}^{2}$ to 1 it means the better fitting.

\section{RESULTS AND DISSCUSSION}

Basic statistical descriptive parameters of three DE granulations (Pura, Super and Ultra) and a perlite granulation, measured by the morphometric method described above, are given in Table 1. The mean diameter value varies from $7.56 \mu \mathrm{m}$ 
Table 1. Basic statistical descriptive parameters of DE and perlite granulations

\begin{tabular}{|l|r|r|r|r|}
\hline & DE Pura & DE Super & DE Ultra & \multicolumn{1}{|c|}{ Perlite } \\
\hline Mean $[\mu \mathrm{m}]$ & 7.56 & 15.27 & 16.64 & 18.72 \\
\hline Max $[\mu \mathrm{m}]$ & 30.00 & 125.00 & 150.00 & 90.00 \\
\hline$R M S[\mu \mathrm{m}]$ & 4.51 & 14.75 & 19.25 & 12.7 \\
\hline Coef. of var. $-C v[\%]$ & 59.62 & 96.59 & 115.72 & 67.87 \\
\hline Skewness [-] & 2.25 & 2.62 & 3.08 & 1.49 \\
\hline Flatness [-] & 8.74 & 13.4 & 15.28 & 6.28 \\
\hline
\end{tabular}

for DE Pura and being $18.72 \mu \mathrm{m}$ for perlite. Corresponding maximum values are 30,125 and 150 $\mu \mathrm{m}$ for DE granulations and $90 \mu \mathrm{m}$ for perlite.

Coefficient of variation $C v$, shows the largest size dispersion of DE Ultra medium around the mean value $(C v=115.72 \%)$. In contrast, the DE Pura $(C v=59.62 \%)$ and perlite $(C v=67.87 \%)$ are more tightly concentrated around the mean values of their diameters, while DE Super, having value of $C v=96.59 \%$ is in the middle with respect to other filter aid material according to the criteria of data dispersion.

Particle size distributions of all tested filter materials are skewed, showing deviations

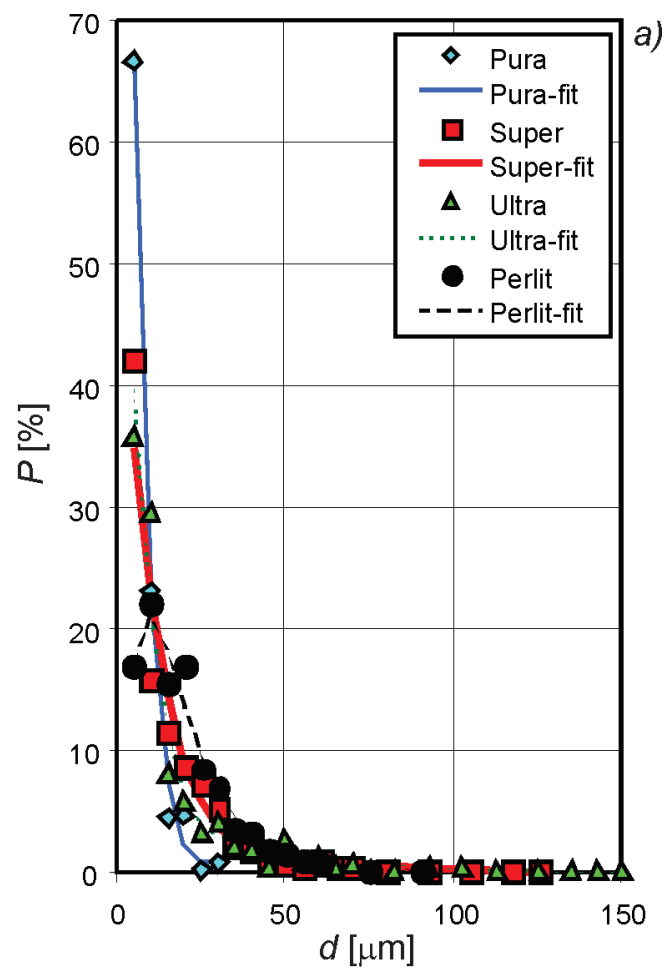

from the normal Gaussian distribution. Skewness factors are always positive, having values of 2.25 , 2.62 and 3.08 for DE granulations Pura, Super and Ultra, respectively, and 1.49 for perlite. Thus, particle size distribution of the perlite granulation is the closest to the symmetrical behavior.

According to the flatness factor, with values of $8.74,13.4,15.28$ and 6.28 for DE Pura, DE Super, DE Ultra and perlite, respectively, perlite granulation is the closest to the normal Gaussian distribution, while the DE Ultra granulation expresses the largest deviation from this function.

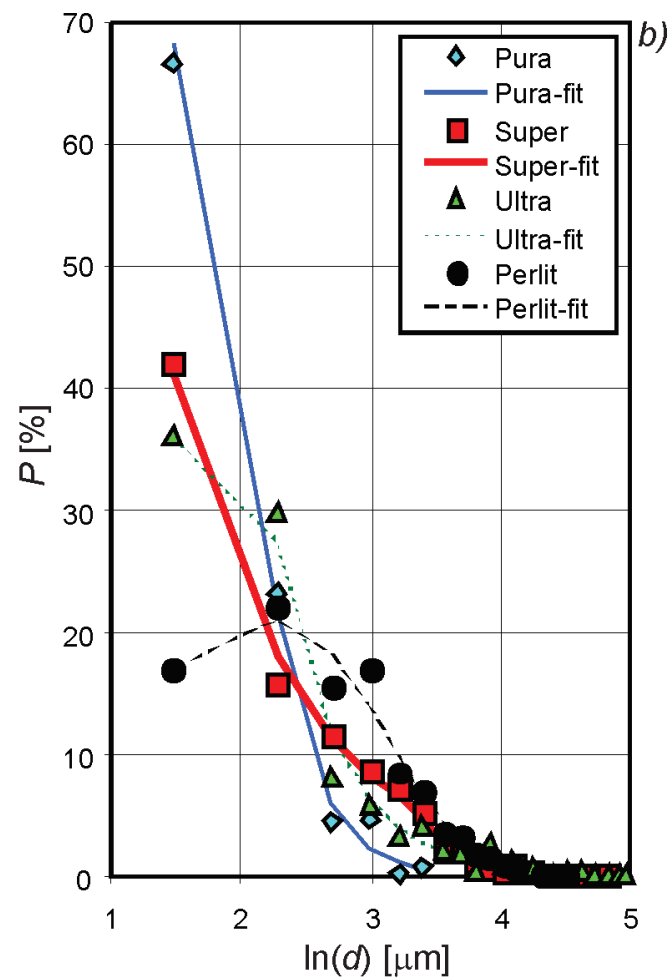

Fig. 2. Fitting results of the particle size distributions of three DE and one perlite granulation; a) hyperbolic function, b) log-hyperbolic function 
Finally, data are fitted using the hyperbolic and log-hyperbolic function. The applied computer program follows algorithm [28]. The resulting data are presented in Fig. 2, where ordinate axis $P[\%]$ represents the empirical and estimated (fitted) values of the percent participation of the granulations particle size classes of diameter $d[\mu \mathrm{m}]$. It is evident that both functions follow experimental data fairly well in all tested cases.

The parameters that characterize the accuracy of fitting procedures are given in Table 2. In the case of DE Pura and perlite, both models provide a nearly identical accuracy, i.e. SSE,
$R M S E$ and $R^{2}$ values are nearly identical. In the case of DE Super and DE Ultra, log-hyperbolic function gave slightly better results - the $R^{2}$ values are higher, while the SSE and RMSE are lower. However, in all tested cases, the $R^{2}$ factors are very high, over 0.93 , which confirms a capability of hyperbolic and log-hyperbolic model to describe particles size distributions of these filter materials. Furthermore, standard errors were up to $2.72 \%$ in all cases, which is acceptable from the practical engineering point of view.

Analytically describing the size distributions of different filtering granulations is a

Table 2. Determination factors of different $D E$ and perlite filter medium particle sizes distributions fitting by hyperbolic and log-hyperbolic functions

\begin{tabular}{|c|c|c|c|c|c|}
\hline & FUNCTION & DE Pura & DE Super & DE Ultra & Perlite \\
\hline \multirow{2}{*}{$S S E[\%]$} & Hyperbolic & 14.65 & 109.77 & 96.74 & 21.95 \\
\cline { 2 - 6 } & Log-hyperbolic & 14.84 & 7.81 & 23.51 & 21.76 \\
\hline \multirow{2}{*}{$R M S E[\%]$} & Hyperbolic & 2.71 & 2.70 & 2.26 & 1.48 \\
\cline { 2 - 6 } & Log-hyperbolic & 2.72 & 0.72 & 1.11 & 1.48 \\
\hline \multirow{2}{*}{$R^{2}[-]$} & Hyperbolic & 0.9956 & 0.9389 & 0.9489 & 0.9707 \\
\cline { 2 - 6 } & Log-hyperbolic & 0.9956 & 0.9957 & 0.9876 & 0.9709 \\
\hline
\end{tabular}
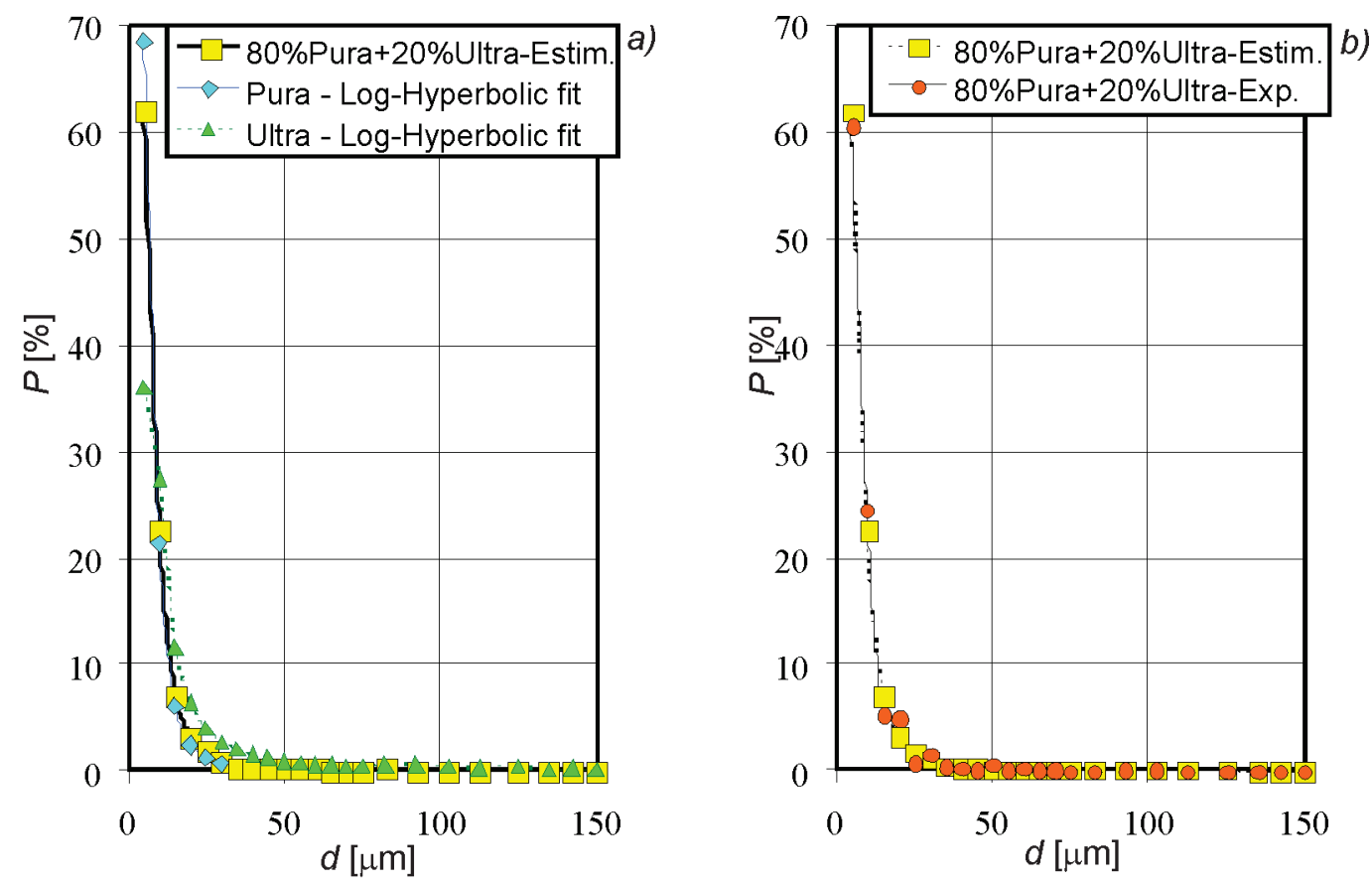

Fig. 3. The particle size distribution of composed granulation consisting of $80 \%$ DE Pura and $20 \%$ DE Ultra; a) particle size distributions of DE Pura, DE Ultra and their composition, predicted by loghyperbolic fit, b) comparison between the experimental and particle sizes distribution of composition predicted by log-hyperbolic fit 
small but important step in developing the models of various filtration processes. Among others, starting from the known particle size distributions of just a few granulations, it is possible to compose a variety of filter mediums. Fig. 3 illustrates the prediction of the size distribution of a composed granulation consisting of $80 \%$ of DE Pura and $20 \%$ of DE Ultra medium, based on the fitting of the particle size distributions of these two materials by log-hyperbolic functions.

Fig. 3a illustrates log-hyperbolic fits of the particle size distributions of DE Pura, DE Ultra and their composition consisting of $80 \%$ DE Pura and $20 \%$ DE Ultra. The particle size distribution of this composition is estimated by combining the log-hyperbolic fitting functions of two parent $\mathrm{DE}$ components. Fig. $3 \mathrm{~b}$ presents a comparison between the experimental and estimated particle size distribution of the composition. It is evident that estimated data agree well with the experimental points, verifying the applicability of the presented approach in predicting and modelling the particle size distributions of granulation compositions.

\section{CONCLUSIONS}

The applicability of the presented experimental and numerical method, in analyzing and describing the particles size distributions of different filter granulation mediums, is verified in the paper. Both the hyperbolic and log-hyperbolic function gave similar fitting results. Still, the log-hyperbolic model has a slight advantage in resolving the problems of this kind. However, a more detailed analysis, based on larger samples of the tested but also of the additional filter granulations of various ranges and produced by various manufacturers, is needed in the future. This will provide more detailed, reliable and accurate results. The other fitting functions should also be tested.

The presented approach facilitates a detailed analysis of granulation filter aid materials. This way, depending on the imposed filtration requirements, a wide variety of different granulations characterised by appropriate particle size distributions can be composed from a few available granulations. The analogue approach can be applied to modelling the particle size distributions of the contaminants born by the fluid that is subjected to filtration, enabling establishing of interrelations between the inlet/outlet fluid parameters and filter granulations particle size distributions.

\section{ACKNOWLEDGMENTS}

This research was supported by the Ministry of Science and Technological Development, Republic of Serbia - projects: OI 174011, TR35035, TR36001.

\section{REFERENCES}

[1] Drev, D., Vrhovšek, D., Panjan, J. (2006). Using porous ceramics as a substrate or filter media during the cleaning of sewage. Strojniški vestnik - Journal of Mechanical Engineering, vol. 52, no. 4, p. 250-263.

[2] Golubović, Z., Šešlija, D., Milovanović, B., Majstorović, B., Vidović, M. (2007). The challenges in sterile pressurised air preparation. Proceedings of the PAMM Conference PC, p. 110-120.

[3] Golubović, Z., Mitrović, Č., Stanojević M. (2004). On the maintenace of filtration systems. The Scientiffical-Technical Journal Research and Design for Economy, vol. 2, no 6, p. 49-56. (in Serbian)

[4] Mitrović, Č., Golubović, Z., Šešlija, D. (2005). Fluid filtartion and separation of hasardeous materials in aircrafts. The Scientiffical-Technical Journal Research and Design for Economy, vol. 3, no. 10, p. 7-20. (in Serbian)

[5] Mitrović, Č., Golubović, Z., Šešlija, D. (2006). Implementation, importance and effetcs of the filtration in economy. The Scientiffical-Technical Journal Research and Design for Economy, vol. 4, no. 12, p. 13-20. (in Serbian)

[6] Van Reis, R., Zydney, A. (2007). Bioprocess membrane technology. Journal of Membrane Science, vol. 297, p. 16-50.

[7] Roberts, L.R., Evans, D., Harris, L. (2009). Removal of TSE agents by depth or membrane filtration from plasma products. Biologicals, doi:10.1016/j.biologicals. 2009. 09.004 . 
[8] Russ, W., Moertel, H., Meyer-Pittroff, R., Babeck, A. (2006). Kieselguhr sludge from the deep bed filtration of beverages as a source for silicon in the production of calcium silicate bricks. Journal of the European Ceramic Society, vol. 26, p. 25472559.

[9] Daufinl, G., Escudier, J.-P., Carrere, H., Berot, S., Fillaudeau, L., Decloux, M., (2001). Recent and emerging applications of membrane processes in the food and dairy industry. Trans. IChemE, vol. 79, part C, p. 89-102.

[10] J. Senèe, J., Robillard, B., Vignes-Adler, M. (1999). Films and foams of champagne wines. Food Hydrocolloids, vol. 13, p. 15-26.

[11] Fillaudeau L., Boissier B., Moreau, A., Blanpain-avet, P., Ermolaev, S., Jitariouk, N., Gourdon, A. (2007). Investigation of rotating and vibrating filtration for clarification of rough beer. Journal of Food Engineering, vol. 80, p. 206-217.

[12] Wegmanna, M., Michen, B., Graule, T. (2008). Nanostructured surface modification of microporous ceramics for efficient virus filtration. Journal of the European Ceramic Society, vol. 28, p. 1603-1612.

[13] Tsai, W.-T., Hsu, H.-C., Su, T.-Y., Lin, K.Y., Lin, C.-M. (2008). Removal of basic dye (methylene blue) from wastewaters utilizing beer brewery waste. Journal of Hazardous Materials, vol. 154, p. 73-78.

[14] Holy, R., Poživil, J. (2002). Batch control system project for a pharmaceutical plant. ISA Transactions, vol. 41, p. 245-254.

[15] Talip, Z., Eral, M., Hiçsönmez, Ü. (2009). Adsorption of thorium from aqueous solutions by perlite. Journal of Environmental Radioactivity, vol. 100, p. 139-143.

[16] Acemioğlu, B. (2005). Batch kinetic study of sorption of methylene blue by perlite. Chemical Engineering Journal, vol. 106, p. 73-81.

[17] Guo-hua, Y., Jiang-hua, Z. (2007). Experimental study on a new dual-layer granular bed filter for removing particulates. Journal of China University of Mining \& Technology, vol. 17, no. 2, p. 201-204.

[18] Stevenson, G.D. (1997). Flow and filtration through granular media - the effect of grain and particle size dispersion. Water Research, vol. 31, no. 2, p. 310-322.

[19] Wakeman, R. (2007). The influence of particle properties on filtration. Separation and Purification Technology, vol. 58, p. 234241.

[20] Tasić, J.S., Golubović, Z.Z., Petrović, V.D., Golubović, Đ.Z. (2009). On the applicability of morphometric method for evaluation of the waterborne particles size distribution. Proceedings of the 26 $6^{\text {th }}$ Symposium on Advances in Experimental Mechanics, p. 227-228.

[21] Žajdela, B., Hriberšek, M., Hribernik, A. (2008). Experimental investigations of porosity and permeability of flocs in the suspensions of biological water treatment plants. Strojniški vestnik - Journal of Mechanical Engineering, vol. 54, no. 7-8, p. 547-556.

[22] Maletić, R. (2005). Methods of statistical analysis. Faculty of Agriculture, Belgrade. (in Serbian)

[23] Emri, I., Cvelbar, R. (2006). Using spline functions to smooth discrete data. Strojniški vestnik - Journal of Mechanical Engineering, vol. 52, no. 3, p. 181-194.

[24] Grešovnik, I. (2007). The use of moving least squares for a smooth approximation of sampled data. Strojniški vestnik - Journal of Mechanical Engineering, vol. 53, no. 9, p. 582-598.

[25] Seber, F.A.G., Wild, J.C. (2003). Nonlinear regression, Wiley \& Sons, New York.

[26] Barndorf-Nielsen, O. (1977). Exponentially decreasing distributions for the logarithm of particle size. Proceedings of the Royal Society, A.353, p. 401-419.

[27] Bartholdy, J., Christiansen, C., Pedersen, B.T.J. (2007). Comparing spatial grain-size trends inferred from textural parameters using percentile statistical parameters and those based on the log-hyperbolic method. Sedimentary Geology, vol. 202, p. 436-452.

[28] Bhatia, J. C., Durst, F. (1988). LHPDF - A PC package for estimating parameters of the log-hyperbolic distribution, moments and mean parameters, Report LSTM 230/T/88, LSTM, Technische Fakultät, Universität Erlangen-Nürnberg, Erlagen. 